\title{
Development of a Reconstruction Methodology Based on X-Ray Computed Tomography to Generate Realistic 3D Concrete Microstructures in MOSAIC
}

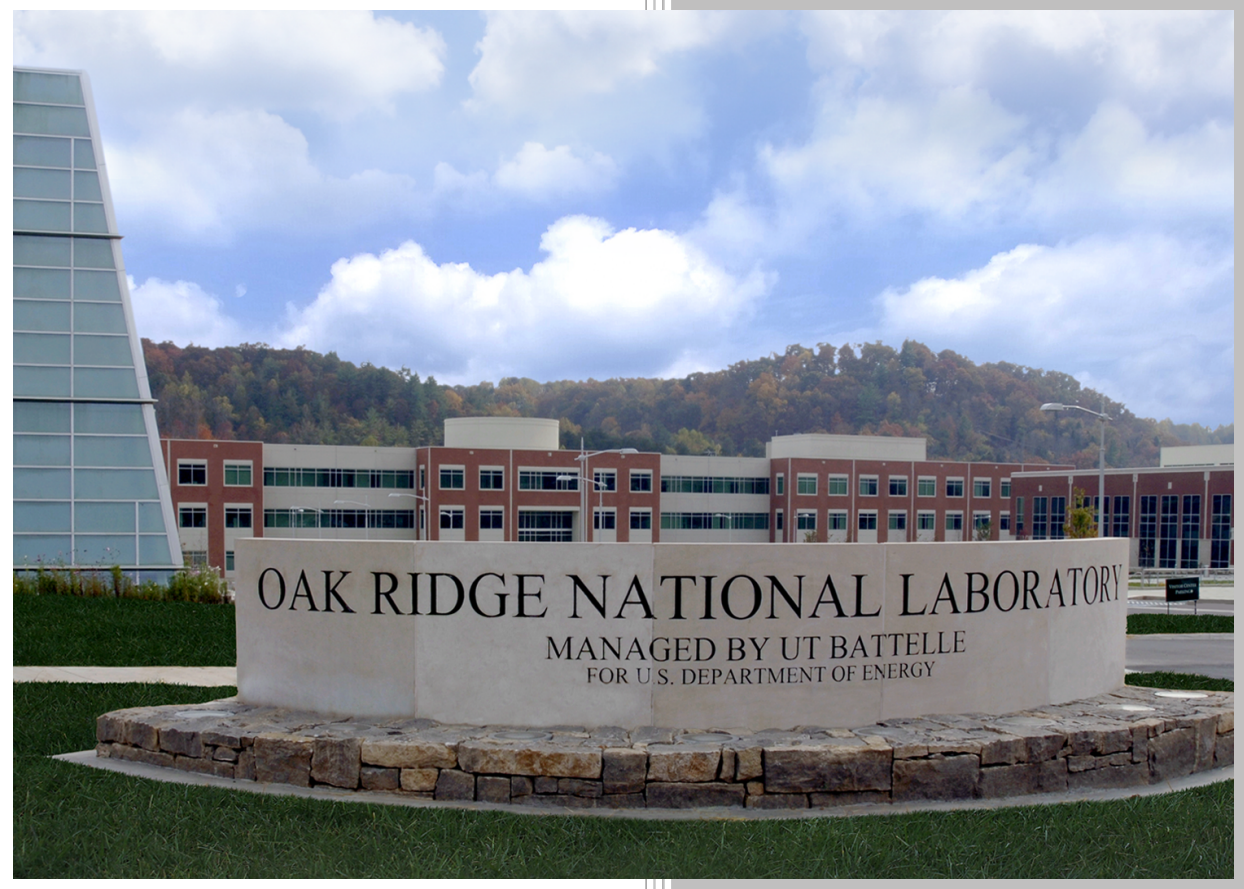

Amani Cheniour

Yujie Li

Yann Le Pape

Ercan Cakmak

J. David Arregui-Mena

Elena Tajuelo Rodriguez

Thomas M. Rosseel

August 2021 


\section{DOCUMENT AVAILABILITY}

Reports produced after January 1, 1996, are generally available free via US Department of Energy (DOE) SciTech Connect.

Website: http://www.osti.gov/scitech/

Reports produced before January 1, 1996, may be purchased by members of the public from the following source:

National Technical Information Service

5285 Port Royal Road

Springfield, VA 22161

Telephone: 703-605-6000 (1-800-553-6847)

TDD: 703-487-4639

Fax: 703-605-6900

E-mail: info@ntis.gov

Website: http://classic.ntis.gov/

Reports are available to DOE employees, DOE contractors, Energy Technology Data Exchange representatives, and International Nuclear Information System representatives from the following source:

Office of Scientific and Technical Information

PO Box 62

Oak Ridge, TN 37831

Telephone: 865-576-8401

Fax: 865-576-5728

E-mail: report@osti.gov

Website: http://www.osti.gov

This report was prepared as an account of work sponsored by an agency of the United States Government. Neither the United States Government nor any agency thereof, nor any of their employees, makes any warranty, express or implied, or assumes any legal liability or responsibility for the accuracy, completeness, or usefulness of any information, apparatus, product, or process disclosed, or represents that its use would not infringe privately owned rights. Reference herein to any specific commercial product, process, or service by trade name, trademark, manufacturer, or otherwise, does not necessarily constitute or imply its endorsement, recommendation, or favoring by the United States Government or any agency thereof. The views and opinions of authors expressed herein do not necessarily state or reflect those of the United States Government or any agency thereof. 


\title{
DEVELOPMENT OF A RECONSTRUCTION METHODOLOGY BASED ON X-RAY COMPUTED TOMOGRAPHY TO GENERATE REALISTIC 3D CONCRETE MICROSTRUCTURES IN MOSAIC
}

\author{
Amani Cheniour \\ Yujie Li \\ Yann Le Pape \\ Ercan Cakmak \\ J. David Arregui-Mena \\ Elena Tajuelo Rodriguez \\ Thomas M. Rosseel
}

Date Published: August 2021

Prepared by

OAK RIDGE NATIONAL LABORATORY

Oak Ridge, TN 37831-6283

managed by

UT-Battelle, LLC

for the

US DEPARTMENT OF ENERGY

under contract DE-AC05-00OR22725 


\section{CONTENTS}

LIST OF FIGURES $\ldots \ldots \ldots \ldots \ldots \ldots \ldots \ldots \ldots \ldots \ldots \ldots$ iv

LIST OF TABLES $\ldots \ldots \ldots \ldots \ldots \ldots \ldots \ldots \ldots \ldots$

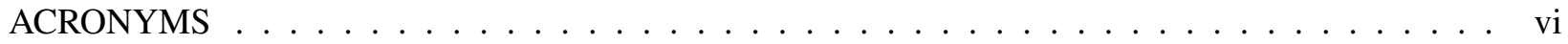

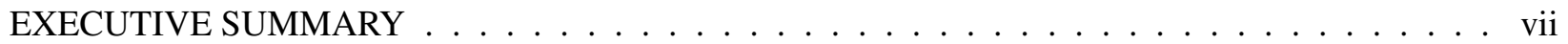

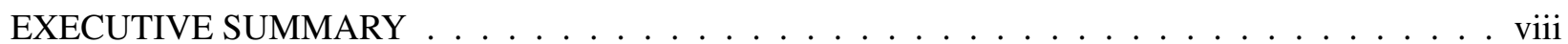

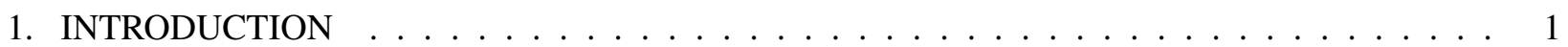

2. XCT-BASED CONCRETE SAMPLE RECONSTRUCTION . . . . . . . . . . . . . 3

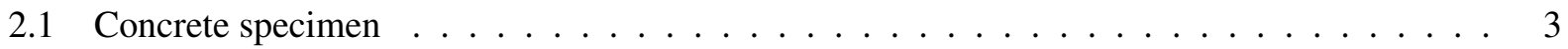

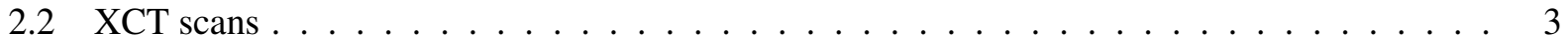

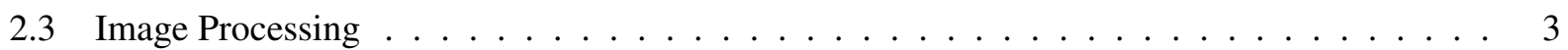

3. IMPLEMENTATION OF A VORONOI STRUCTURE IN AGGREGATE PHASES . . . . . . . . 7

$3.13 \mathrm{D}$ Mesh Generation $\ldots \ldots \ldots \ldots \ldots \ldots$

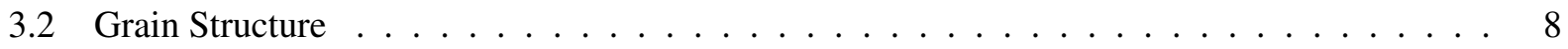

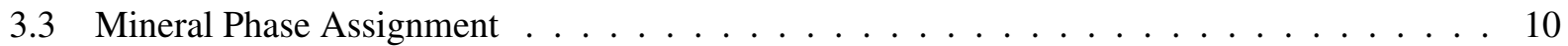

4. GENERATION OF 3D PHASE MAPS IN MOSAIC . . . . . . . . . . . . . . . . . 12

4.1 Gradient-Based Particle Maps . . . . . . . . . . . . . . . . . . . . . . 12

4.2 Example 3D Simulation in MOSAIC . . . . . . . . . . . . . . . . . 13

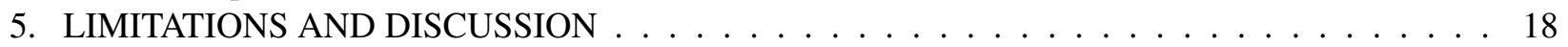

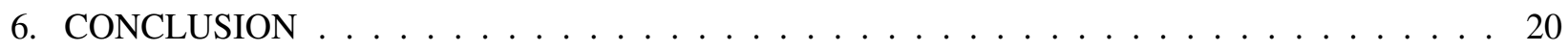

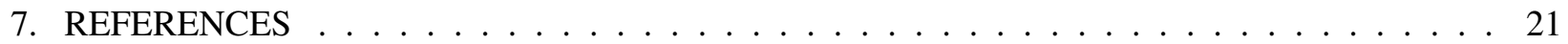




\section{LIST OF FIGURES}

1 Example XCT-based reconstructed concrete image. . . . . . . . . . . . . . 4

2 Example gray scale histogram of an XCT-based image of Con-A. . . . . . . . . . . 5

3 Image processing steps. . . . . . . . . . . . . . . . . . 6

4 Average distance between each data point and its 30 nearest neighbors in ascending order. . . 7

5 FE block assignment based on gray level in the input image. . . . . . . . . . . . 8

$6 \quad 3$ D mesh blocks (half concrete sample). . . . . . . . . . . . . . . . . . 9

7 Generation of a grain structure to represent aggregate particles in the concrete half-sample. . 10

8 Example grains (red) attributed to a single order parameter in the aggregate phase. . . . . . . 11

9 Example hcp gradient map of a single layer of the 3D structure. . . . . . . . . . . . . . 12

10 Three consecutive layers from the generated phase map: quartz (red), albite (orange), biotite (yellow), anorthite (sky blue), microcline (green), clinochlore (royal blue), IPI (black), ITZ (blue), hcp (light gray), and void (purple) . . . . . . . . . . . . . . . . 13

11 MOSAIC VTK output of the 3D microstructure . . . . . . . . . . . . . . . . . 14

12 Clipped 3D domain (a) and the corresponding stress magnitude (b) at nine days of irradiation. 16

13 Total strain (a) and creep strain (b) in clipped 3D domain at nine days of irradiation. . . . . . 17 


\section{LIST OF TABLES}

1 Phase volume fractions $\ldots \ldots \ldots \ldots \ldots \ldots \ldots$

2 Linear viscoelasticity model parameters . . . . . . . . . . . . . . . . . . 14 


\author{
ABBREVIATIONS \\ CBS concrete biological shield \\ DBSCAN density-based spatial clustering of applications with noise \\ FEM finite element method \\ FFT fast-Fourier transform \\ HCP hardened cement paste \\ IMAC irradiated minerals, aggregate and concrete \\ IPI interparticle interface \\ ITZ interfacial transition zone \\ JCAMP Japan Concrete Aging Management Program \\ LWRS Light Water Reactor Sustainability \\ MOOSE Multiphysics Object-Oriented Simulation Environment \\ MOSAIC Microstructure-Oriented Scientific Analysis of Irradiated Concrete \\ mXRF micro X-ray fluorescence \\ NRC Nuclear Regulatory Commission \\ ORNL Oak Ridge National Laboratory \\ RIVE radiation-induced volumetric expansion \\ XCT X-ray computed tomography
}




\section{ACKNOWLEDGEMENTS}

This work was sponsored by the US Department of Energy Office of Nuclear Energy's Light Water Reactor Sustainability (LWRS) Program under contract DE-AC05-00OR22725 with UT Battelle LLC/Oak Ridge National Laboratory. 


\section{EXECUTIVE SUMMARY}

Despite being passive components, concrete structures represent a major capital investment in nuclear power plants. However, some concrete components are critical for the safety and long-term operation of the reactors: the concrete containment building protects the reactors from external aggression. The concrete biological shield (CBS) contains the radiation exiting the reactor to protect equipment and personnel. Depending on the design, the CBS also has a function for supporting the reactor's systems. This function needs to be ensured in-service and during accident scenarios. After several decades of use, the CBS is exposed to high-energy irradiation over an extended operation time, exceeding the fluence level currently considered detrimental for the concrete's structural properties. Such conditions occur at different times depending on the reactor's operation and design. Nevertheless, from the perspective of subsequent licence application, it is mandatory to assess the potential effects of prolonged irradiation on concrete.

Unlike steel components, concrete structures are built with local constituents (cement, sand, and coarse aggregates) to avoid prohibitive transportation costs. Thus, concrete performance is determined by the tolerance or the susceptibility of its constituents against any aging mechanism. The tolerance against irradiation of the CBSs may vary greatly among the 56 nuclear power plants and 93 nuclear power reactors that form the current US light-water reactor fleet. Irradiation-induced degradation is mainly a result of amorphizationinduced expansions of the silicated minerals present in the aggregates. The neutron irradiation-induced expansion varies from one rock-forming mineral to another: well framework-structured silicates (e.g., quartz and feldspars) exhibit higher swelling than chained (e.g., pyroxene) or isolated silicates (e.g., garnet). Silicates make up nearly $90 \%$ of the Earth's crust. Other common minerals present in construction aggregates are carbonates found, for example, in limestone. Irradiated carbonates exhibit only very minor expansions.

Because the rates and amplitudes of radiation-induced expansion vary considerably among the aggregateforming minerals, the mismatch expansion can cause cracking in irradiated aggregates and thus a degradation of mechanical properties. Additionally, the overall expansion of the aggregates creates important mechanical energy storage in the cement paste. The stored energy dissipation occurs either by viscous relaxation or cracking, the latter contributing to further loss of the concrete's structural properties. The interactions among the degradation mechanisms involved during concrete irradiation are quite complex, time dependent, highly nonlinear, and highly dependent on the mineralogy of the concrete aggregates. For these reasons, an effective modeling strategy must be implemented at the mineral scale. The Light Water Reactor Sustainability (LWRS) program has been developing an integrated approach that combines advanced characterization methods and fast-Fourier transform (FFT)-based nonlinear simulations. The MicrostructureOriented Scientific Analysis of Irradiated Concrete (MOSAIC) tool provides a rigorous approach to analyzing concrete specimens. It employs physics-based models to account for the effects of irradiation, temperature, damage, and creep, which are the primary factors in the degradation process.

The development and validation activities of MOSAIC-2D were documented in previous LWRS reports and publications, including the following:

- Y. Li, A. Cheniour, Y. Le Pape, E. Tajuelo Rodriguez, Validation of 2D-MOSAIC Tool for Assessment of Concrete Sensitivity to Aging-Induced Damage Using the Existing Concrete Properties Database, ORNL/SPR-2021/1912, March 2021;

- Y. Li, Y. Le Pape, E. Tajuelo Rodriguez, C.E. Torrence, J. D. Arregui Mena, T. M. Rosseel, and M. Sircar. "Microstructural Characterization and Assessment of Mechanical Properties of Concrete Based on Combined Elemental Analysis Techniques and Fast-Fourier Transform-Based Simulations." Con- 
struction and Building Materials, 257, Oct 2020. ISSN 09500618. doi: 10.1016/j.conbuildmat.2020.119500. https://linkinghub.elsevier.com/retrieve/pii/S0950061820315051.

- Christa E. Torrence, Alain B. Giorl, Yujie Li, Elena Tajuelo Rodriguez, Jose D. Arregui Mena, Thomas M. Rosseel, Yann Le Pape. "MOSAIC: An Effective FFT-Based Numerical Method to Assess Aging Properties of Concrete." Journal of Advanced Concrete Technology, 192, pp. 149-167, Released February 27, 2021, Online ISSN 1347-3913, https://doi.org/10.3151/jact.19.149, https: //www.jstage.jst.go.jp/article/jact/19/2/19_149/_article/-char/en

As detailed in the LWRS report ORNL/SPR-2021/1912, the validation of MOSAIC-2D is largely completed. However, continued validation work will be performed through the collaborative activities of the European Union project ACES to extend MOSAIC's capabilities to other mechanisms including creep, alkali-silica reaction, and delayed ettringite formation.

The development and validation of MOSAIC-3D must be pursued to overcome some of MOSAIC-2D's limitations. In the CBSs of LWRs, the stress state caused by radiation-induced volumetric expansion (RIVE) is highly three-dimensional because of the fluence gradient. Thus, damage is mainly governed by structural constraints caused by the biaxial compression loading near the reactor cavity. 2D simulations led to an overly conservative loss of mechanical properties because of the premature percolation of damage-forming fractures. Therefore, expanding MOSAIC capabilities to perform realistic and predictive 3D simulations is necessary. The development and validation progress is as follows:

1. Implement 3D mechanical simulation capabilities. Task completed in FY20.

2. Develop a reconstruction methodology combining x-ray computed tomography (XCT) and 2D highresolution images to generate realistic concrete aggregates' 3D microstructures. Task scheduled for FY21.

3. Complete the reconstruction of high-resolution 3D concrete microstructures. Task scheduled for FY22.

4. Complete validation of 3D-MOSAIC on irradiated concrete. Task tentatively scheduled for FY22.

This report documents the 3D microstructure generation using XCT. This process follows the following steps:

1. A clustering algorithm is applied to XCT-based images to detect clusters of pixels with similar features and separate the three phases: aggregates, cement paste, and pores/background.

2. The processed images are stacked to form a 3D simulation domain. A finite element method (FEM)based simulation environment, MOOSE, is used to separate the three phases into mesh blocks and generate a grain structure using a Voronoi diagram.

3. The data obtained from the FE code are processed to assign minerals to the newly generated grains inside the aggregate block. Interfaces between particles and between phases are added in MOSAIC, and a full 3D phase map is produced.

A 3D simulation in MOSAIC of irradiated concrete using linear elasticity and RIVE, as well as creep models using the 3D phase map, exemplifies the current level of achievement.

This methodology allows for the generation of a 3D concrete microstructures with good accuracy, which is enabled by the optimization of several parameters used in the image processing step. The methodology 
is effective and incurs a relatively low computational cost for a 3D problem. The current limitations of the proposed approach are related mainly to the 3D characterization's reliance on high-energy $\mathrm{x}$-ray and neutron radiation. In this research, the level of contrast between the concrete constituents phases remains relatively low, making the separation of the phases a rather difficult task. The next research milestone will focus on improving the $3 \mathrm{D}$ characterization to produce a more realistic representation of the aggregates and their forming minerals. 


\section{INTRODUCTION}

The safety and sustainability of nuclear power plants require rigorous assessment of potential irradiationinduced damage in the structural components. The concrete biological shield (CBS) is subjected to neutron and gamma radiation throughout its lifetime, which develops damage with the increasing irradiation dose and can lead to failure. The Microstructure-Oriented Scientific Analysis of Irradiated Concrete (MOSAIC) code was developed by the Light Water Reactors Sustainability (LWRS) program to model the mechanical behavior of concrete under irradiation. MOSAIC has been used successfully and validated in 2D on previously irradiated concrete and aggregate samples ([1] and ORNL/SPR-2021/1912). In 2D, MOSAIC uses realistic high-resolution $(<\approx 50 \mu \mathrm{m})$ microstructures reconstructed from the outputs of a combination of varied techniques -including $\mathrm{x}$-ray diffraction, micro $\mathrm{x}$-ray fluorescence (mXRF), and energy-dispersive $\mathrm{x}$-ray spectroscopy (EDXS) elemental maps collected from the surface of a sample. This combined experimental and modeling strategy enables a significant improvement in the accuracy of the modeled domain, and it incurs relatively low computational cost compared to traditional methods such as the finite element method (FEM). However, the results of such simulations assume a uniform elongated microstructure along a sample's height, which is not representative of the complex shape and composition of its various constituents. More specifically, 2D representations lead to overly conservative estimates of the loss of engineering properties in degraded concrete. This results from the early percolation of damaged areas in the 2D microstructures. In addition, MOSAIC-2D representation does not allow for modeling the complex 3D stress states that develop in nuclear power plants' concrete structures. For example, the highly irradiated belt line region of the CBS in the vicinity of the reactor cavity is subjected to biaxial compression in the vertical and azimuthal directions and traction in the radial direction. This specific 3D stress state is caused by the radiation-induced volumetric expansion (RIVE) and the effects of structural restraints in the three directions.

To address these limitations, research activities were initiated in 2018 to develop 3D capabilities in MOSAIC (LWRS report: ORNL/SPR-2020/1676). The existing 2D constitutive models were extended to 3D, and they have been successfully tested on randomly generated 3D domains. The remaining challenge is obtaining realistic 3D concrete microstructures based on 3D characterization of concrete specimens. Although varied techniques can be employed for surfaces by relying on optical, electron, or x-ray interactions, the main difficulty with $3 \mathrm{D}$ characterization is the simultaneous need for deep penetration (on the order a few centimeters in concrete) and high resolution. This constraint limits the options to high-energy radiation techniques such as X-rays and neutrons. This work focuses on the possibility of using X-ray computed tomography (XCT) data to create 3D MOSAIC-compatible phase maps of concrete.

$\mathrm{XCT}$ is a nondestructive characterization technique that measures the x-ray attenuation with matter at different angles to produce a high-resolution, 3D-voxelized reconstruction of the internal structure of a sample by distinguishing areas of different radiodensities. XCT outputs can be read as a series of 2D highresolution images that represent a specified slice in the sample. In this work, a hollow plain concrete cylinder (d. $40 \mathrm{~mm}$ ) fabricated for an NRC-sponsored research project on irradiated reinforced concrete was used as a test specimen. This specimen was XCT scanned in 2019 for quality control before being shipped to the irradiation test reactor.

The XCT-based sample layers consist of a series of gray scale images of the concrete. The images reveal low contrast between aggregates and the hardened cement paste (HCP), which complicates phase identification through simple segmentation methods such as the application of a threshold to the gray levels. Additionally, the image quality and contrast limitations do not distinguish particles that form the aggregates. 
To address this problem, an artificial Voronoi grain structure was added in the aggregates phase. An image processing methodology was developed to identify three main components in the images - aggregates, HCP, and pores or background - and to generate a Voronoi structure in the aggregates. This report details the application of various numerical tools to produce a realistic 3D phase map in MOSAIC based on the provided XCT scan. 


\section{XCT-BASED CONCRETE SAMPLE RECONSTRUCTION}

\subsection{Concrete specimen}

The concrete specimen used in this study was fabricated for an NRC-sponsored research project on the effects of irradiation on the bond strength of steel embedded in concrete. The concrete formulation strictly follows the specifications from the Japan Concrete Aging Management Program (JCAMP). The concrete specimen has the same phase composition as concrete Con-A described in the paper by Maruyama et al. (2017) [2]: $w / c=0.5$ using a high early strength cement $\left(\rho_{c}=3.14 \mathrm{~g} \mathrm{~cm}^{-3}\right)$ (Taiheiyo Cement Corp.), land sand, sandstone $\left(\rho_{s}=2.61 \mathrm{~g} \mathrm{~cm}^{-3}\right)$ (Shizuoka Prefecture), and altered crushed tuff aggregate, namely $\mathrm{GA} / \mathrm{F}$ from Aichi prefecture. The mix design is as follows: cement, $366 \mathrm{~kg} \mathrm{~m}^{-3}$; water, $183 \mathrm{~kg} \mathrm{~m}^{-3}$; fine aggregate/sand, $799 \mathrm{~kg} \mathrm{~m}^{-3}$; and coarse aggregates, $995 \mathrm{~kg} \mathrm{~m}^{-3}$. The aggregates were composed mostly of quartz (92\%) and feldspars (7\%). Based on the composition data, the volume fraction of aggregates and $\mathrm{HCP}$ are, respectively, $\approx 70 \% / 30 \%$. The average mineral grain size in the aggregates is 0.1 to $0.3 \mathrm{~mm}$ according to [2]. All materials were sourced from Japan, and the specimens were prepared at The University of Tennessee, Knoxville. The cylindrical hollow specimen has a $4 \mathrm{~cm}$ diameter and height. The cylindrical hole at the center of the sample has a diameter of $8 \mathrm{~mm}$. Of the set of fabricated specimens, A-D- 8 was set aside as a "cold" control specimen; it is considered in this research as a representative concrete specimen for the $3 \mathrm{D}$ reconstruction.

\subsection{XCT scans}

An XCT scan of the concrete specimen was performed. The specimen was characterized using Oak Ridge National Laboratory (ORNL) x-ray tomographer ZEISS Xradia 520 Versa. The experiment was conducted at $140 \mathrm{kV}$ with a magnification of $\times 0.4$. The scan completed in about 2.5 hours. The scan generated a reconstructed specimen in a TXM file format. Then, transverse slices of the reconstructed specimen were obtained using the Python library DXchange, which can read a TXM file and convert it to a series of TIFFformatted images. The TIFF format retains high quality and is generally used for large or high-resolution images. The initial image resolution in the slices was $1024 \times 1004$. 1,018 images were generated from the reconstructed sample data. Each image shows a cross section of the hollow cylindrical specimen in addition to the air background, as shown in Figure 1. This section documents the phase identification process used for this specimen to separate aggregate, HCP, and void phases using a Python script. The same process was applied for all the images used in this work. Note that this process can be used for other concrete samples with proper adjustments of the parameters used in image processing.

\subsection{Image Processing}

Initial processing is necessary to facilitate the detection of aggregates within the concrete sample as a result of the low contrast between aggregates and the HCP. In fact, Figure 2 shows that it is difficult to separate aggregates from the HCP using a threshold method because their gray levels are similar. First, the Gaussian blurring method in the Python library OpenCV is used, with the goal of reducing noise within aggregates in the image. The kernel size for the Gaussian filter is $5 \times 5$. The original and resulting images are shown in Figure 3.a and Figure 3.b. An automatic adjustment of contrast and brightness is then applied, and its 


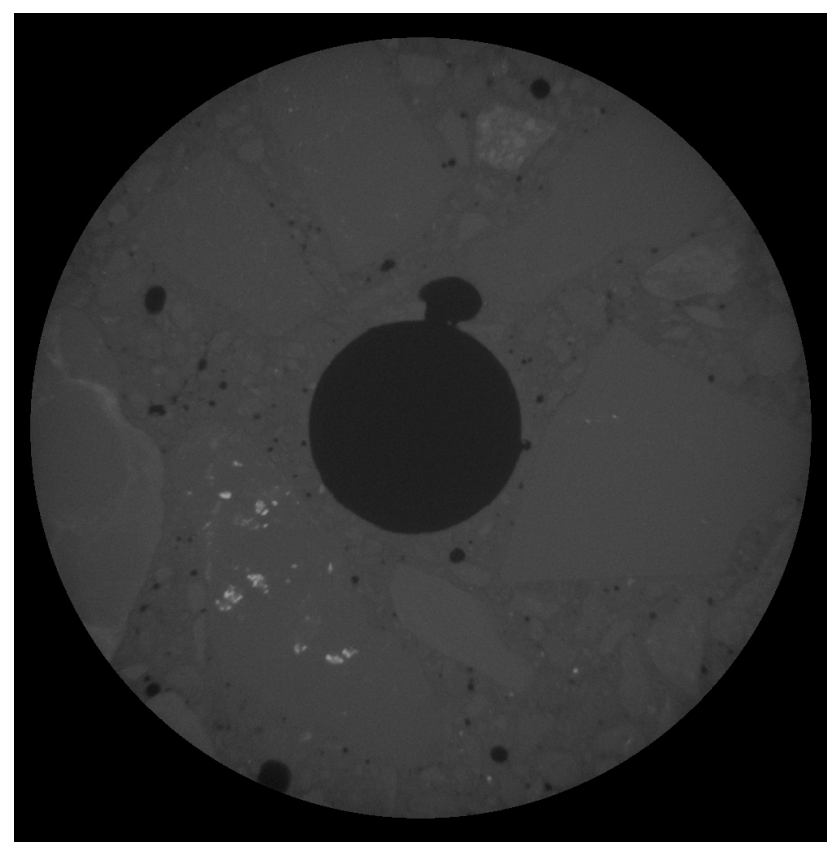

Figure 1. Example XCT-based reconstructed concrete image.

result is shown in Figure 3.c. To determine the location of pixels associated with voids, a binary image is generated based on a specified threshold, as demonstrated in Figure 3.d.

Density-based spatial clustering of applications with noise (DBSCAN) is an unsupervised learning algorithm applied to detect clusters of samples in a dataset, as described by [3]. The algorithm begins by randomly placing an arbitrary point $P_{0}$ and detecting the density of samples around $P_{0}$. For this purpose, two DBSCAN parameters are necessary: (1) the minimum number of samples $k$ (including $P_{0}$ ) in the neighborhood of $P_{0}$ to consider it as a core point, and (2) the maximum distance $\epsilon$ between two samples to be considered neighbors. The distance can be calculated as the Euclidean distance between two samples. Once the algorithm stops detecting nearby samples, it considers the ensemble of detected samples including $P_{0}$ as a cluster; then it generates another point $P_{1}$ and repeats the same process until no more remaining samples can be assigned to a cluster. Boundary points around the cluster are assigned. If a sample does not belong to any cluster at the end of the clustering process, then it is considered noise. Therefore, DBSCAN can be used to segment data, and more specifically, images. Several other clustering and image segmentation algorithms such as k-means and the watershed algorithm are available.

Because of the low contrast and the large difference between cluster or aggregates sizes, DBSCAN was chosen for this work. In fact, it is easier for DBSCAN to separate clusters of different densities; therefore, it is expected to perform well with concrete XCT images. Because the image resolution is high, the application of a density-based clustering algorithm is computationally expensive. Thus, the resolution was reduced by a factor of four in each direction to $256 \times 251$ before segmenting the image. It is theoretically possible to apply DBSCAN to a 3D image; however, it is very difficult in practice because of the sharp increase in computational time. DBSCAN was therefore applied to each 2D image separately using the same parameter values through the free Python library scikit-learn. The distance parameter was set to 4.5, and the minimum number of samples was set to 30 . For visualization purposes, each cluster was assigned a color, and a gray 


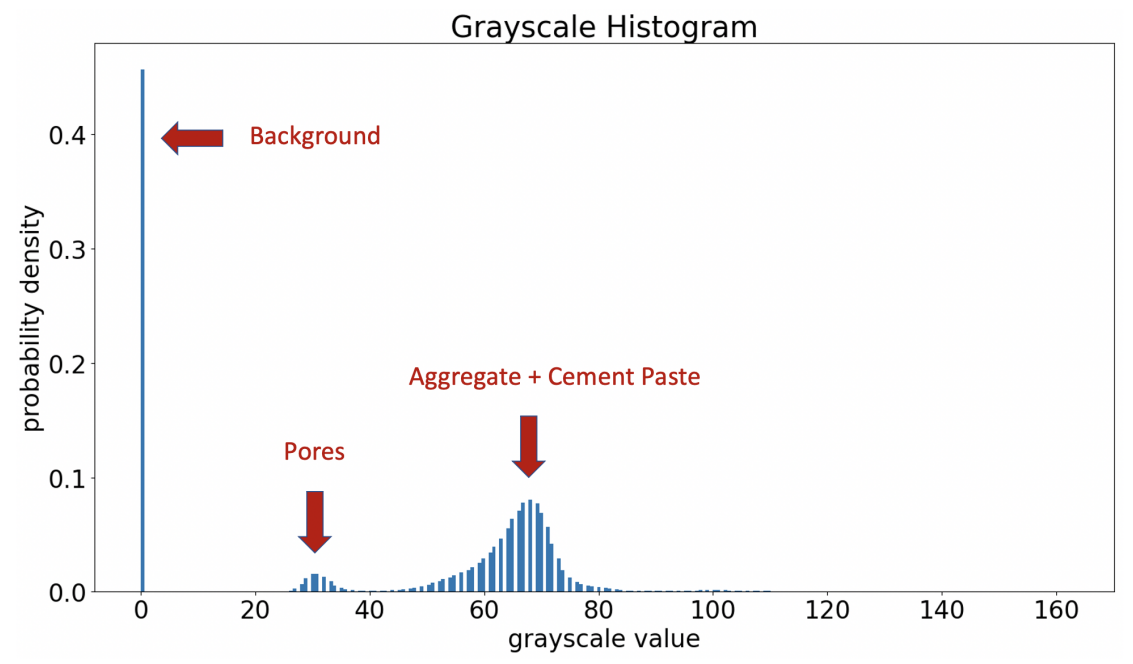

Figure 2. Example gray scale histogram of an XCT-based image of Con-A.

color map was used, as shown in Figure 3.e. The figure shows that the algorithm is capable of detecting large aggregates when using the chosen DBSCAN parameters. It also considers the hole in the middle of the sample as a cluster, in addition to all the pores seen in black in Figure 3.d. To solve this issue, all pixels associated with pores or voids in the image were assigned the value of the background pixel in the segmented image.

The choice of the maximum distance parameter is motivated by the plot of the average distance between each data point and its 30 nearest neighbors in ascending order, as shown in Figure 4 and as suggested in the paper by [4]. The number of nearest neighbors $k$ is a parameter selected through a trial and error process. Figure 4 shows that an $\epsilon$ distance of 4.5 is within the "elbow" zone of the plot and reduces overfitting. This process was repeated for a number of layers to ensure that values chosen for both $\epsilon$ and $k$ produced fairly consistent results.

The DBSCAN output produced clusters that in some cases did not have clearly defined boundaries, as shown in Figure 3.e. Therefore, the last step separated aggregates from each other by applying dilation and then erosion to the image. The kernel size for dilation and erosion is $3 \times 3$. Figure 3.f shows the final image output obtained. Aggregates are shown in different gray levels, and the HCP is white. The void and pore areas are slightly darker than the HCP. Note that the large pore on the left of Figure 3.d is of the same color as the background in the final result. Additionally, the pore/void phase was assigned the same color in all of the processed images for consistency. The phase identification process used in this report is expected to perform better, with improved contrast between aggregates and the HCP. The result shown here is nevertheless satisfactory, given the quality of the original image. 
a. Original

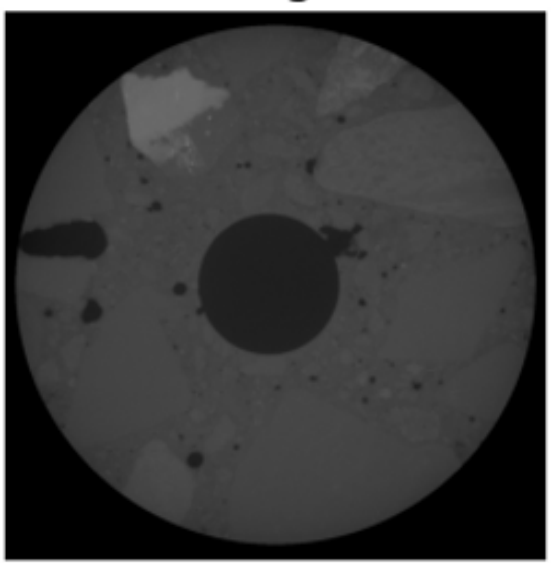

c. Contrast + Brightness

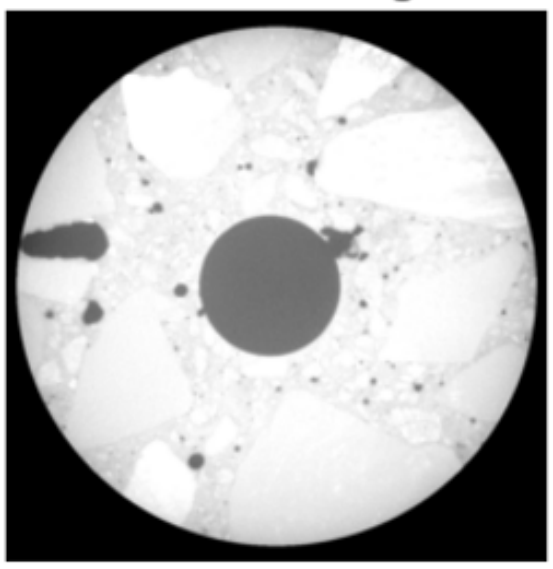

e. DBSCAN

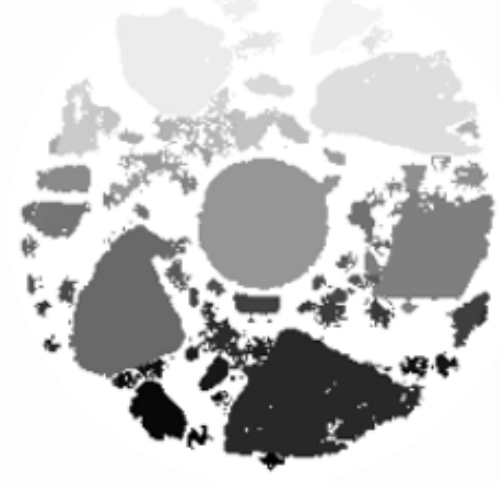

b. Gaussian Blur

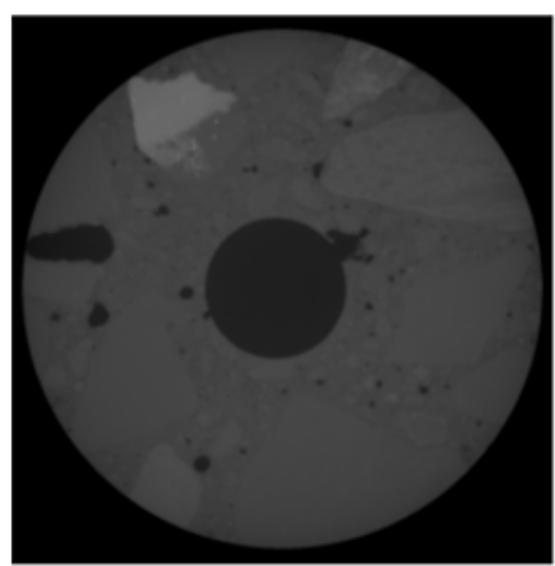

d. Threshold

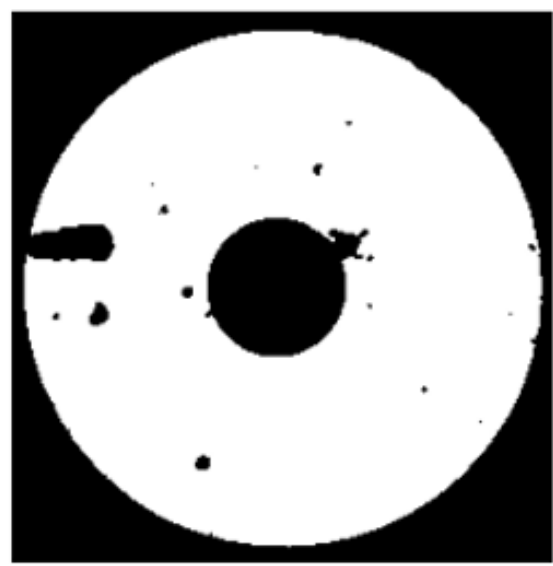

f. Erosion + Dilation

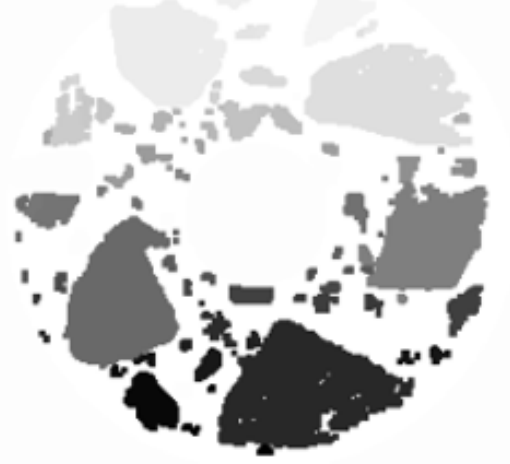

Figure 3. Image processing steps. 


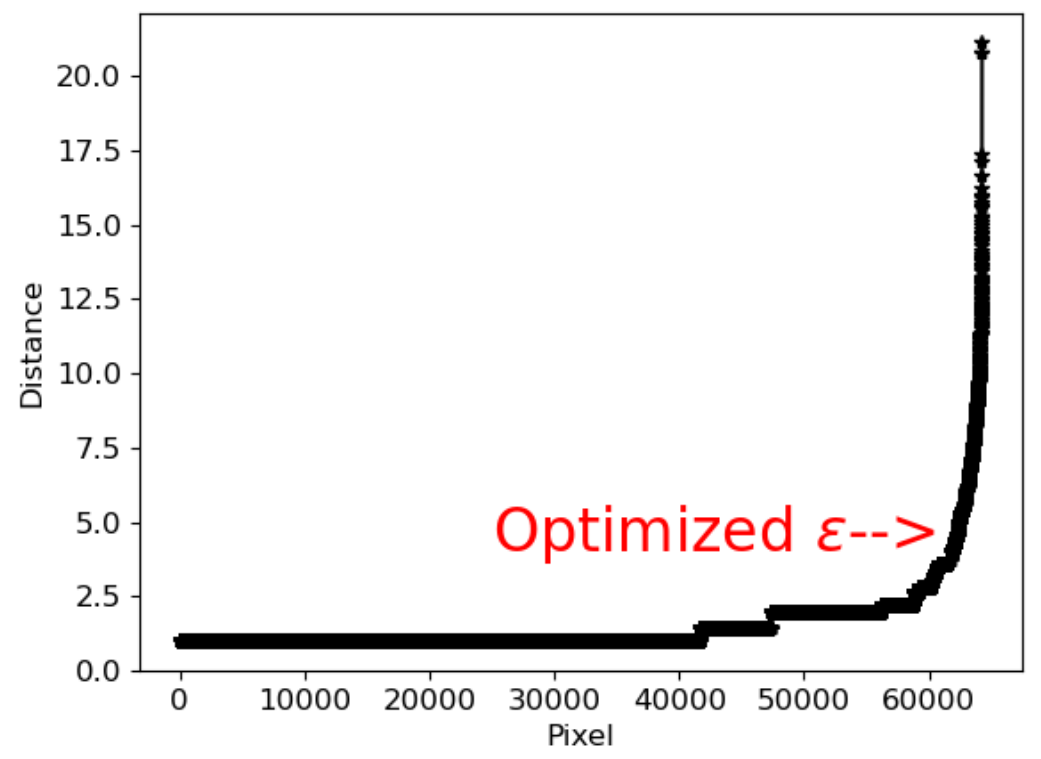

Figure 4. Average distance between each data point and its 30 nearest neighbors in ascending order.

\section{IMPLEMENTATION OF A VORONOI STRUCTURE IN AGGREGATE PHASES}

After the separation of the three main phases - aggregate, HCP, and pores/background — an artificial particle Voronoi structure is implemented in the aggregate phase. The purpose of this step is to generate representative particles and interparticle interfaces (IPI) that separate particles of the same or different mineralogies. In fact, it is not possible to separate particles within the aggregates for two reasons: (1) the contrast is generally low between particles, and (2) the aggregate particles in this concrete specimen are very small, which requires a higher image resolution to detect. This section presents the computational tools used to create 3D particles within the aggregate phase using a Voronoi diagram.

\subsection{D Mesh Generation}

The Multiphysics Object-Oriented Simulation Environment (MOOSE) framework [5] can generate a 3D grain structure using a Voronoi tesselation at a relatively low computational cost and was therefore used in this work. Note that Python libraries that generate comparable structures exist; other methodologies can be explored for this purpose, as well. Because MOOSE is a FEM-based code, a 3D mesh based on the processed images is required to apply the Voronoi tessellation algorithm to the concrete stack of images. The processed images were converted to gray scale images in Python, as shown in Figure 5a, and then read by MOOSE using the ImageSubdomainGenerator tool. This tool allows for the conversion of each generated cluster (from DBSCAN) in the image to an FE block based on its gray level, as shown in Figure $5 \mathrm{~b}$ where each color corresponds to a separate mesh block. To generate the mesh, the element size was set to the pixel size.

MOOSE can also read a stack of images to create a 3D mesh using the color-based generated blocks. The RenameBlockGenerator tool is then used to merge all of the aggregate blocks into a single large block. 


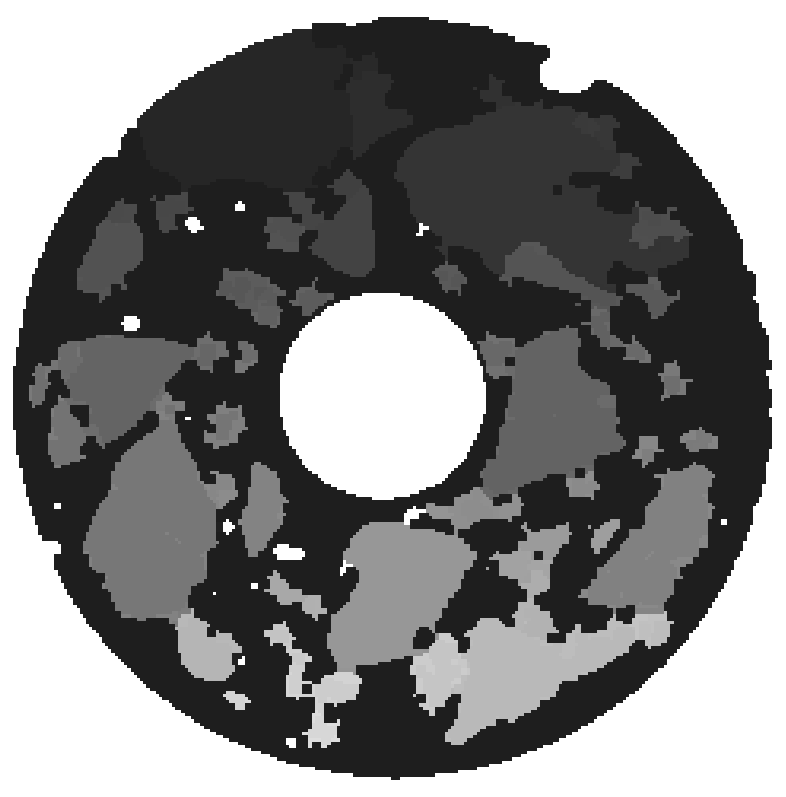

(a) Grayscale image

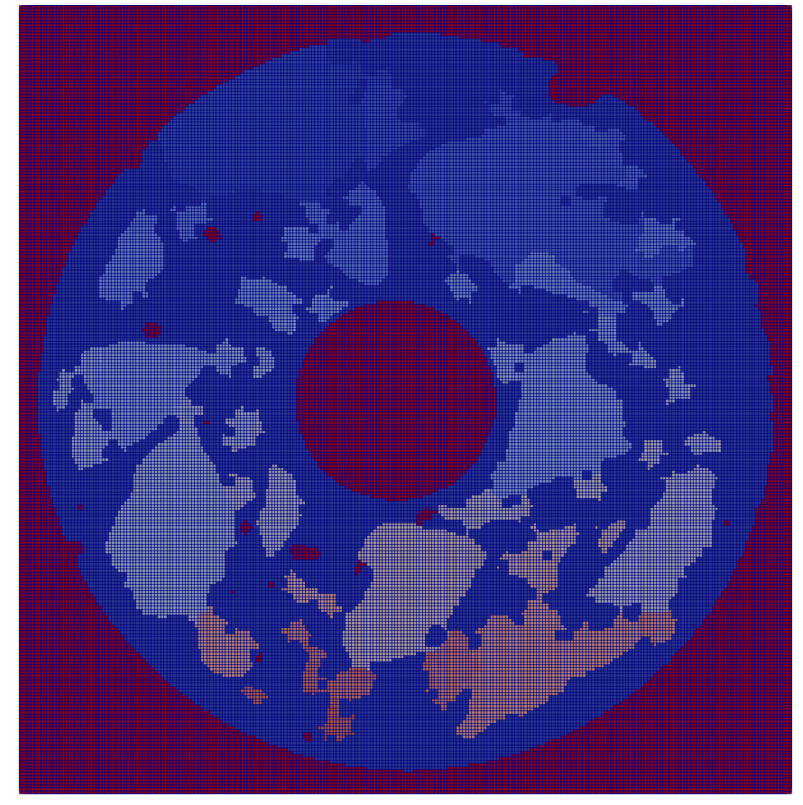

(b) FE mesh blocks

Figure 5. FE block assignment based on gray level in the input image.

Both the HCP and void phases have a unique gray level throughout all of the processed images; therefore, a single 3D block is automatically generated in MOOSE for each of the two phases. The XCT scan generated 1,018 images, from which 509 consecutive images - corresponding to half the concrete sample — were used in this work. The chosen images range from the $201^{\text {st }}$ to the $709^{\text {th }}$ image of the XCT scan. The element size in the vertical direction is calculated based on the height $(2 \mathrm{~cm})$ and the pixel size in the 2D images. The numbers of elements in the $\mathrm{x}, \mathrm{y}$, and $\mathrm{z}$ directions are $N_{x}=251, N_{y}=256$, and $N_{z}=121$, respectively. Because $N_{z}$ is smaller than the number of images (509), several images were stacked into a single (x-y) element layer along the vertical (z) direction. The resulting 3D structure is shown in Figure 6, in which each color corresponds to a unique block.

\subsection{Grain Structure}

The PolycrystalVoronoi tool in the UserObject block of a MOOSE input file allows for the generation of a grain structure, where a Voronoi tessellation can be performed based on randomly placed seeds across the computational domain. For simplicity, the grains were generated across the entire domain; however, the grain data were extracted from the aggregate block only in postprocessing, and this was done for visualization purposes only. Grains are stored in variables called order parameters, which are equal to 1 inside the grain and equal to 0 outside the grain. A smooth interface can be applied for better solve convergence. MOOSE can associate multiple grains to a single variable to eliminate the need for one variable per grain, which significantly reduces the computational costs for problems with a large number of grains.

For the application described herein, one thousand grains were generated inside the 3D structure in Figure 6. In practice, a significantly larger number of grains will be needed to represent the very fine 


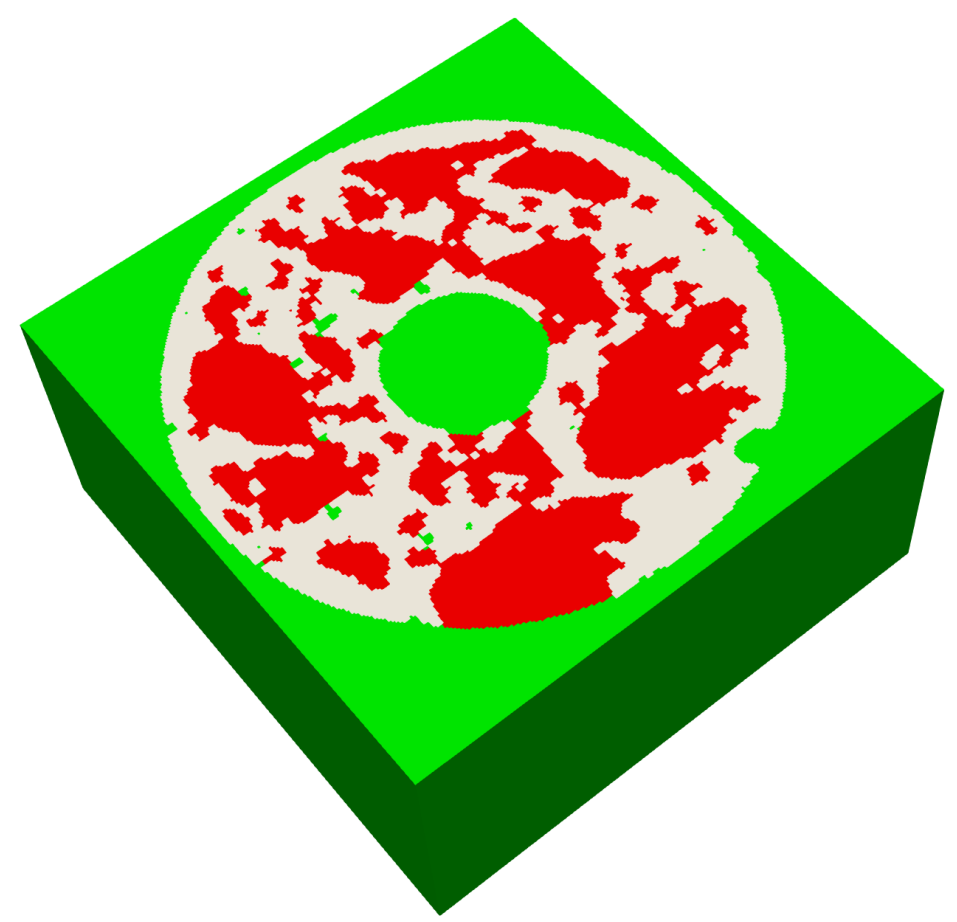

Figure 6. 3D mesh blocks (half concrete sample).

aggregate particles in Con-A, which is very computationally expensive. It is therefore recommended to use smaller concrete samples if the application of interest requires the generation of very fine grains. For the purpose of demonstrating the process of developing 3D phase maps for MOSAIC simulations, it is sufficient to assume the particle size to be larger than the real average particle size in Con-A. For improved visualization, the void and background phases are hidden in the following illustrations: Figure 7 shows the 3D grain structure in the aggregate block surrounded by the HCP block (Figure 7a) and separated from the HCP block (Figure 7b). Note that the pores and void volumes seen in Figure 7a, as well as the aggregate block in Figure 7b, smoothly transition from one element to another in the z-direction as a result of a fairly consistent result of both the binary threshold and the DBSCAN outputs along the XCT-based layers of images.

The DistributedRectilinearMeshGenerator mesh tool in MOOSE assigns a copy of a part of a mesh to each processor instead of copying the entire mesh. In other words, the mesh is generated in parallel, and the number of partitions created corresponds to the number of processor units used in the simulation. Since MOOSE uses Message Passing Interface capabilities to run simulations in parallel, it is capable of using multiple nodes to construct a mesh in parallel. This is very beneficial for 3D simulations with large grain numbers, which typically require 25 order parameters or more-and thus much more memory usage. This parallel capability is therefore used in this work. Additionally, memory usage is further reduced by using the nemesis output type, which consists of multiple mesh partition files. The number of output files also corresponds to the number of processors requested. The output files can be read easily as a single batch in the visualization software ParaView [6]. For this work, 30 order parameters were requested to generate 1,000 grains using 72 processors distributed over 2 nodes. The final memory usage was approximately 164 GB. With an interfacial width slightly larger than a single element, only 15 variables were ultimately used 


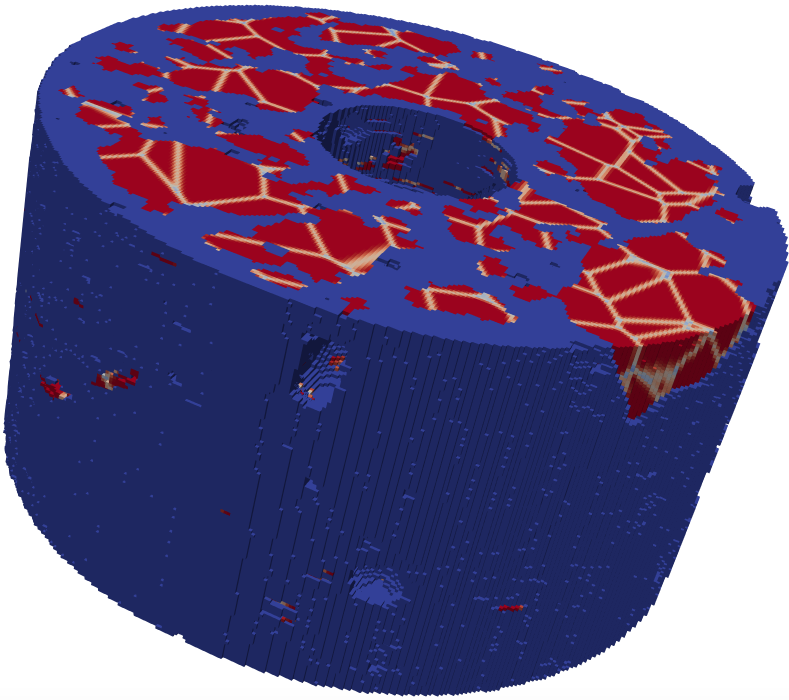

(a) Cement paste and aggregate phases

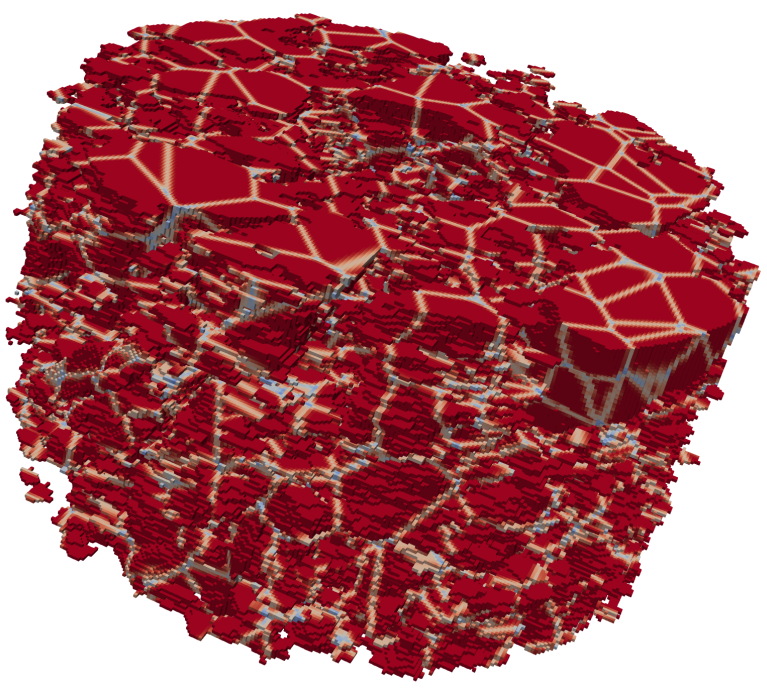

(b) Aggregate phase

Figure 7. Generation of a grain structure to represent aggregate particles in the concrete half-sample.

by the simulation. The choice of the thin interface width facilitates the generation of the one-pixel-width IPI phase at a later step in MOSAIC.

Finally, the constructed 3D mesh is read and converted to a voxelized structure using the Resample To Image filter in ParaView. A CSV data file is then exported from the voxelized structure. This file contains the calculated values of each variable or order parameter in each generated voxel, as well as other information such as the block ID and the element or voxel ID. In addition to the grain variables or order parameters, an auxiliary variable $B n d s$ is defined such that

$$
\text { Bnds }=\sum_{i} G r_{i}^{2}
$$

where $G r_{i}$ is the order parameter corresponding to grain $i$, and Bnds is calculated using BndsCalcAux in MOOSE in the 3D domain to determine the location of grain boundaries. Bnds is equal to 1 inside grains and is less than 1 at grain boundaries.

\subsection{Mineral Phase Assignment}

As stated above, each variable is assigned several grains throughout the 3D structure, as shown in Figure 8. First, the volume fraction occupied by each order parameter is evaluated to approximately match the composition of the Con-A aggregates ( $92 \%$ quartz, 3\% microcline, 2\% anorthite, and less than 1\% for each of chlorite, biotite, orthoclase, anorthoclase, and albite as mentioned in [2]). Then, a number of order parameters is assigned a mineral such that the resulting volume fraction of the mineral is close to the experimental phase composition. A specific phase is assigned if the block ID corresponds to the aggregate block and if both the order parameters $G r_{i}$ and Bnds are higher than a set threshold (0.6 in this work). A voxel map of each phase is then generated such that if the latter conditions are met, then a constant value different from 0 is weighted by Bnds to produce gradients around interfaces. Note that another possible method to reduce 


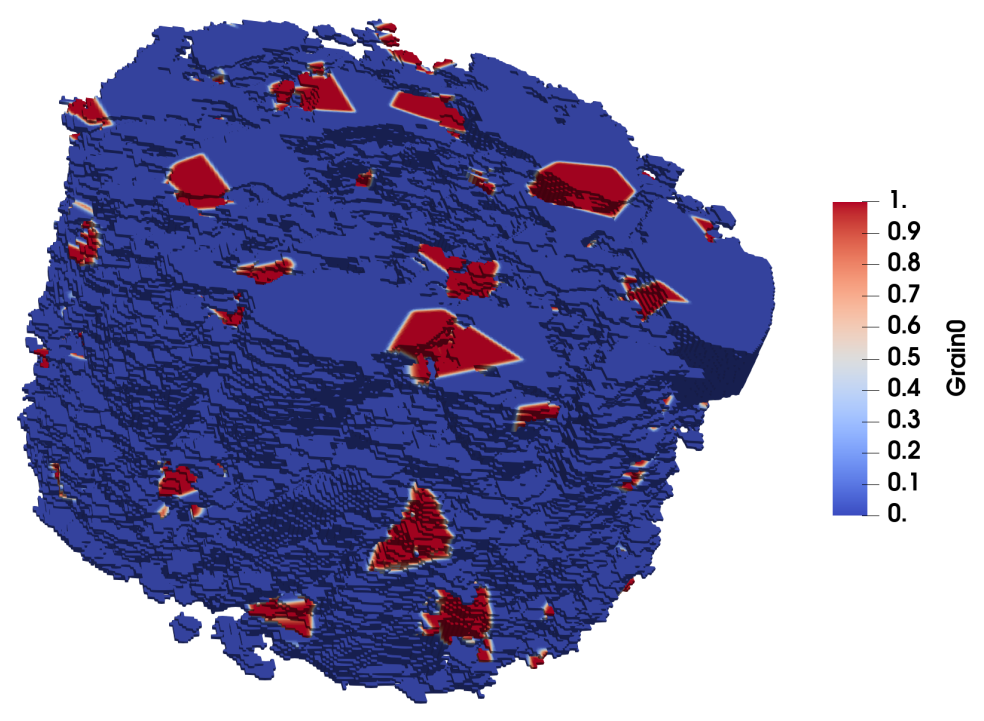

Figure 8. Example grains (red) attributed to a single order parameter in the aggregate phase.

the error in the volume fractions is to assign a phase to each grain—instead of each variable—by isolating the grains in postprocessing.

For simplicity, the number of phases was reduced in this work. Chlorite is represented by clinochlore. The average particle size in $3 \mathrm{D}$ is $0.54 \mathrm{~mm}$ (calculated by assuming spherical particles) and the aggregate volume fraction is approximately $50 \%$. A CSV file containing a $30976 \times 251$ matrix is generated for each phase. The matrix contains the "mirrored" data to accommodate MOSAIC's axis direction choices. 


\section{GENERATION OF 3D PHASE MAPS IN MOSAIC}

\subsection{Gradient-Based Particle Maps}

In order to generate a compatible phase map in XML format, MOSAIC uses a gradient-based approach to determine the location of each phase. The previously generated CSV files of different phases are imported via a MOSAIC input script and converted to normalized weights. The gradient map of each phase is generated as shown in Figure 9, and the maximum gradient is used to generate a seed map for the watershed algorithm implemented in the code. The watershed algorithm creates a particle map. In each particle, an average composition is calculated based on the data in the imported CSV files. If the fraction of a given phase is higher than a specified threshold, then the particle is assigned to the phase. MOSAIC then generates an initial phase map in XML format. In this phase map, the IPI and the interfacial transition zone (ITZ) are grouped into a single "boundaries" group of voxels. Therefore, another MOSAIC script is necessary to assign either the IPI or the ITZ identity to each boundary voxel.

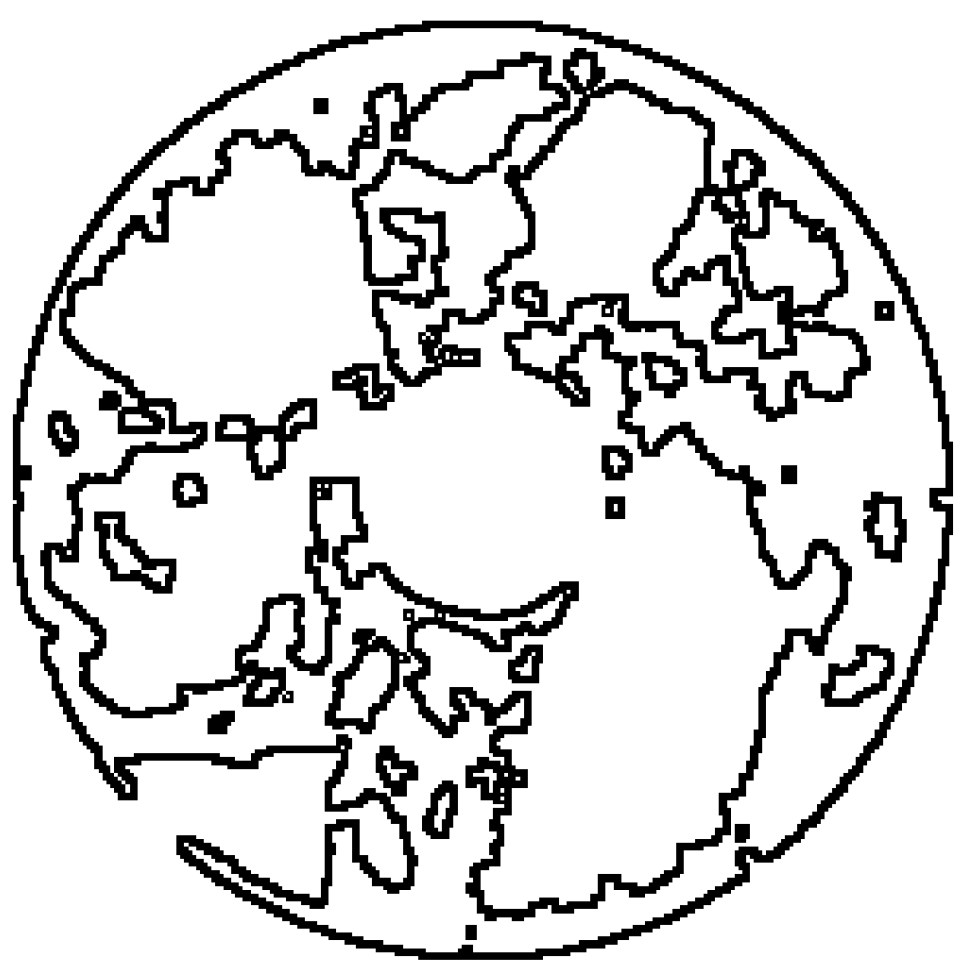

Figure 9. Example hcp gradient map of a single layer of the 3D structure.

Using a second MOSAIC script, voxels separating a matrix phase from the rest of the phases in the initial phase map are identified as ITZ. The matrix phase is set to HCP. The remaining boundary voxels represent the IPI. The final volume fractions are listed in Table 1. Figure 10 shows 3 out of 121 layers of the 3D structure using the minerals listed in Table 1, interfaces, HCP, and void. Note that the boundary between HCP and pores/void is considered as ITZ for simplicity. However, it is possible to separate it from the ITZ using a contour detection method in Python, which, for instance, can be applied to the binary images obtained from the threshold image in Figure 3.f. The MOSAIC script generates a complete 3D phase map 
in XML format. The phase map can be used to model the mechanical behavior of the reconstructed concrete sample in MOSAIC.

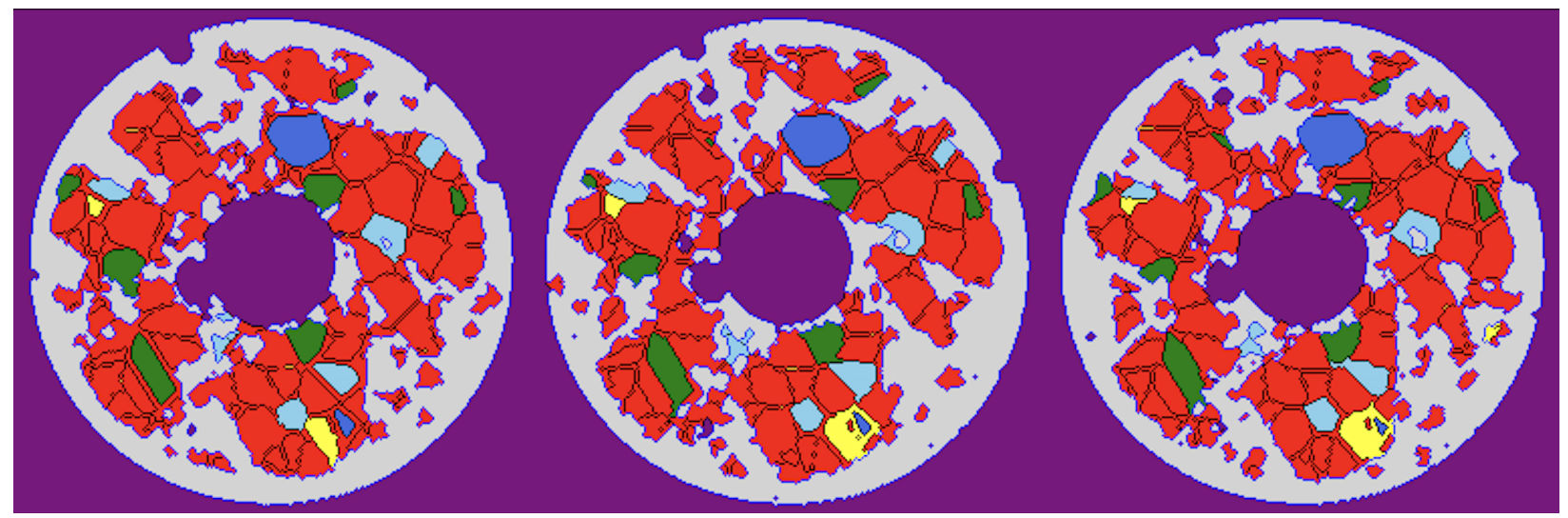

Figure 10. Three consecutive layers from the generated phase map: quartz (red), albite (orange), biotite (yellow), anorthite (sky blue), microcline (green), clinochlore (royal blue), IPI (black), ITZ (blue), hcp (light gray), and void (purple).

Table 1. Phase volume fractions

\begin{tabular}{c||c|c|c|c|c|c|c} 
Phase & Quartz & Microcline & Anorthite & Albite & Biotite & Clinochlore & IPI \\
\hline Volume fraction [\%] & 76.85 & 3.76 & 3.38 & $<0.1$ & 1.97 & 0.52 & 13.47
\end{tabular}

\subsection{Example 3D Simulation in MOSAIC}

The phase map is generated by stacking 121 2D layers, which creates a 2D domain of $\left(N_{y} \times N_{z}\right) \times N_{x}$. The dimensions parameter of the phase map is manually changed to $N_{z} \times N_{y} \times N_{x}$ to apply constitutive models in 3D. To test the 3D phase map, a simple irradiated concrete model in MOSAIC is used. All six minerals and the IPI are subjected to isotropic RIVE. For minerals, RIVE and elastic properties are taken directly from the irradiated minerals, aggregate and concrete (IMAC) database [7]. The linear elasticity model accounts for the anisotropy of minerals. The IPI's Young's modulus is $101.15 \mathrm{GPa}$, and its Poisson's ratio is 0.079, based on Voigt-Reuss bounds approximations in Con-A. The IPI's RIVE model and parameters are also determined using homogenization techniques based on the minerals' volume fractions determined experimentally and their respective RIVE models. The HCP Young's modulus is $12 \mathrm{GPa}$, and its Poisson's ratio is $0.2[8]$.

The ITZ's Young's modulus is set to 4.5 GPa with the same Poisson's ratio as HCP. In both the ITZ and HCP, a generalized Kelvin-Voigt model of linear viscoelasticity is used to model creep. The creep model parameters are summarized in Table 2. A linear elasticity model is applied to the void phase, assuming a small enough Young's modulus to still allow for smooth convergence of the solve while not significantly affecting the mechanical behavior of the sample. The void's Young's modulus is set to $0.1 \mathrm{GPa}$, and its Poisson's ratio is set to 0.2 .

To reduce the computational cost of the simulation, the model does not include damage development and propagation. The temperature is set to the average temperature $\left(66.7^{\circ} \mathrm{C}\right)$ in the Con-A specimen with 
Table 2. Linear viscoelasticity model parameters

\begin{tabular}{c||c|c} 
Phase & HCP & ITZ \\
\hline Creep modulus (GPa) & 15 & 5 \\
Creep viscosity (days) & $0.1,3$ & $0.1,3$
\end{tabular}

the highest irradiation dose (PPT-E in [2]) and the flux is set to $4.2198 \times 10^{-3} \mathrm{n} / \mathrm{pm}^{2} /$ day. Free boundary conditions are applied in all three directions. The linear and nonlinear mechanics solvers' tolerances are set to $2 \times 10^{-4}$ and $1 \times 10^{-3}$, respectively. The simulation domain shown in Figure 11 is extracted in Visualization Toolkit (VTK) format, which is compatible with ParaView. The stress, total strain, and creep strain fields are also extracted at each time step. Figure 12 shows the clipped 3D image of the simulation domain in Figure 12a and the corresponding stress norm or magnitude in Figure $12 \mathrm{~b}$ after approximately nine days of irradiation.

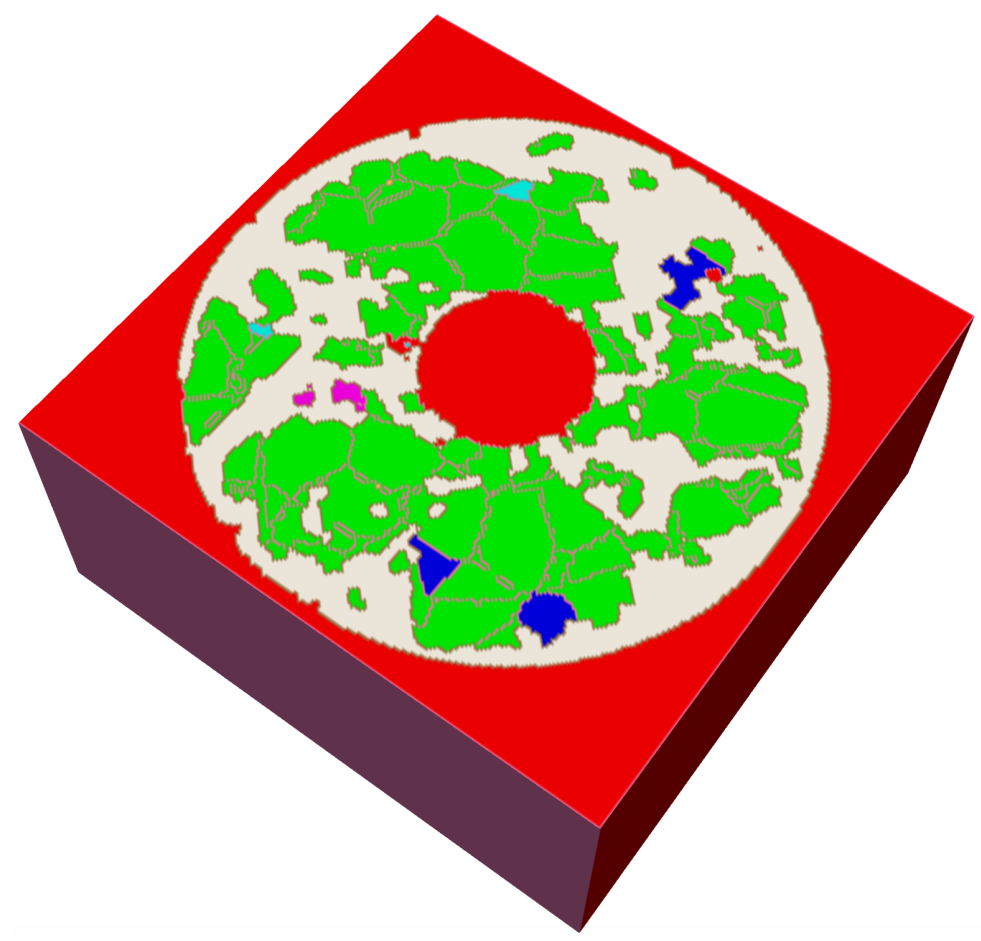

Figure 11. MOSAIC VTK output of the 3D microstructure.

Figure 11 shows that the aggregate composition is mostly homogeneous, as a result of the dominant quartz volume fraction shown in green (VTK-generated color). Other minerals are found in smaller proportions, such as biotite in pink and microcline in blue. Figure 12 demonstrates the impact of the large isotropic expansion of quartz under irradiation on other minerals present in the aggregates, and it shows that the stress magnitude is the highest inside those minerals. The clipped 3D domains also reveal some discontinuities in the aggregates or HCP phases along the vertical directions as a result of (among other reasons) the processing of 2D images independently of each other, as described in Section 2.3. Although such discontinuities create a certain error in the simulated mechanical behavior of the sample, the overall result is rather good and satisfactory given the initial low contrast between phases in the reconstructed XCT 
images of the concrete sample.

Figure 13 shows both the total strain and the creep strain fields in the clipped 3D domain shown in Figure 12a. Figure 13a shows that quartz particles have a higher total strain than other aggregate particles, which is consistent with the stress field shown in Figure 12b. Figure 13b shows the creep strain in HCP and ITZ phases. The creep strain is the highest at sharp interface regions between the HCP and aggregates.

The results of this 3D simulation are consistent with the chosen simplicity of the irradiated concrete model. However, the presence of pores and voids phases should be treated carefully to avoid nonphysical mechanical behavior in the 3D domain. In fact, Figure 13a shows that despite the artificial low void's Young's modulus, the total strain is not negligible in this phase. 


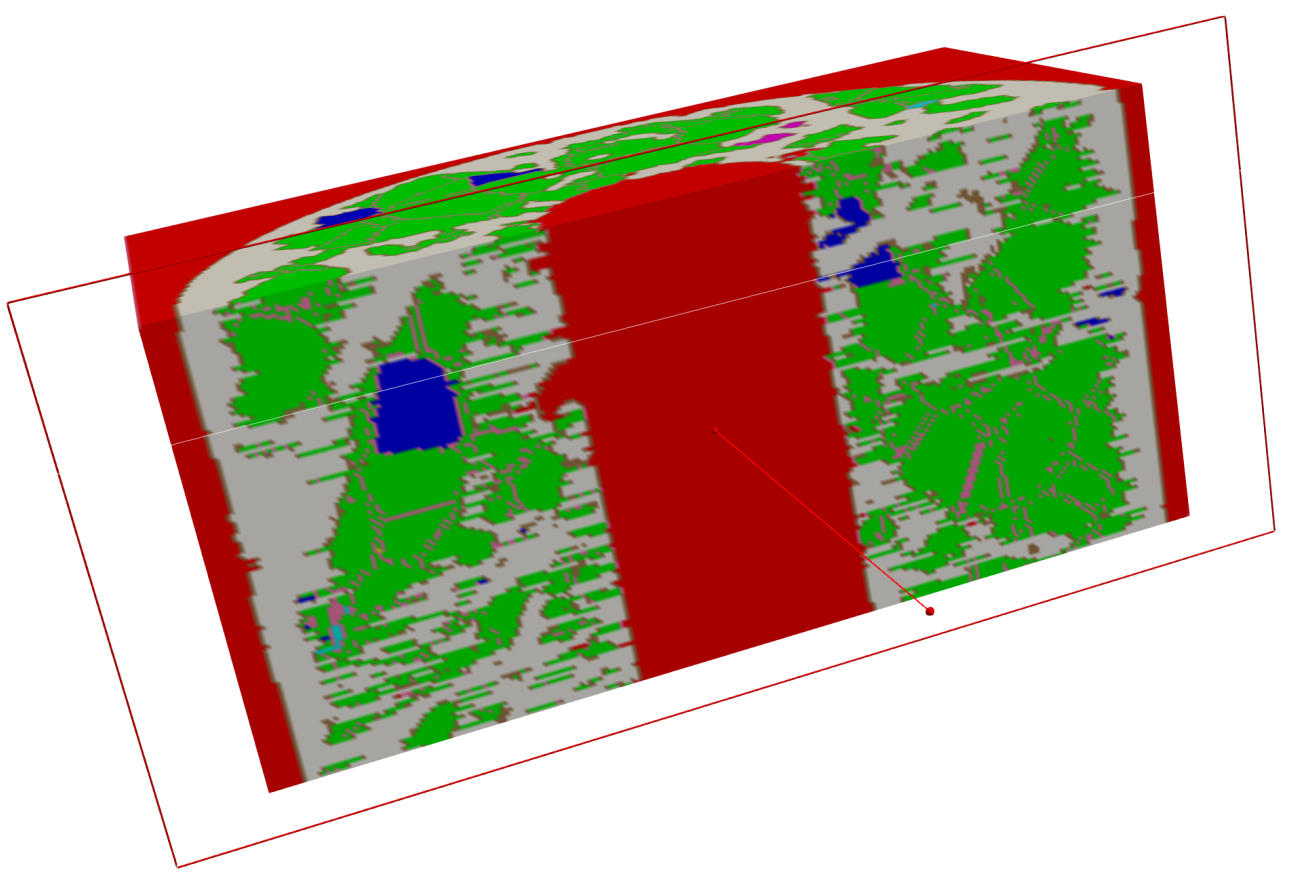

(a) Clipped 3D domain.

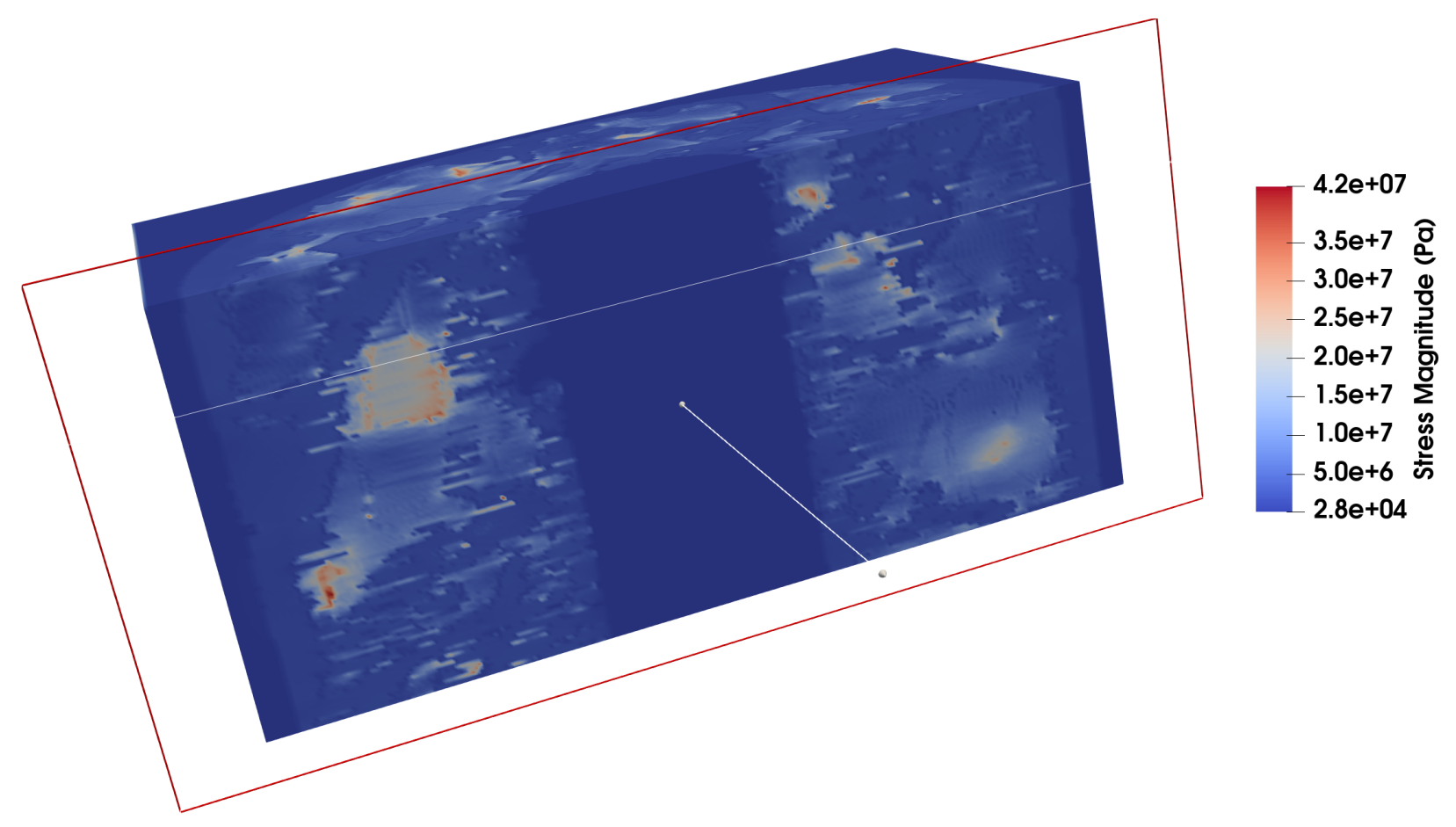

(b) Stress magnitude.

Figure 12. Clipped 3D domain (a) and the corresponding stress magnitude (b) at nine days of irradiation. 


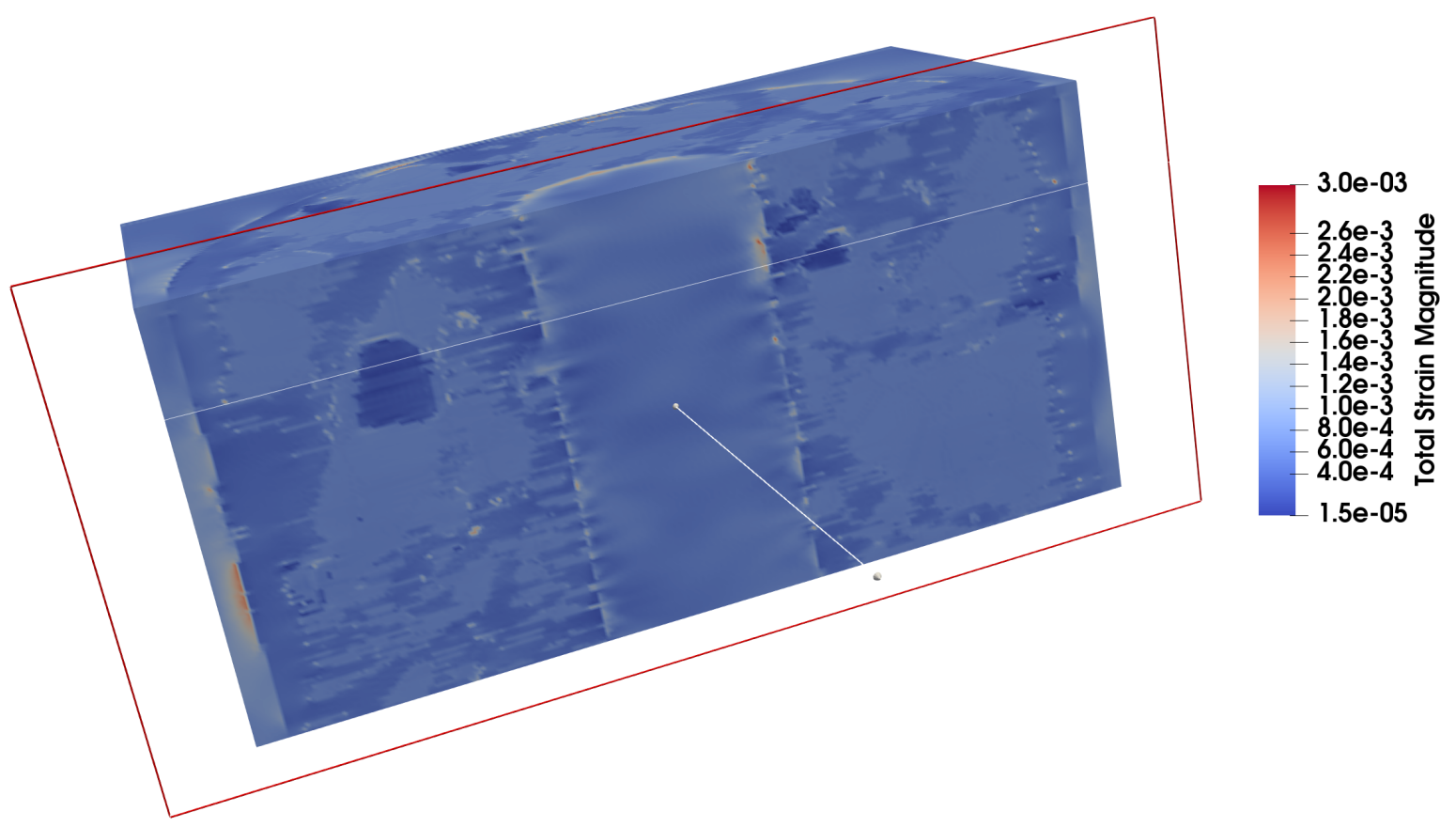

(a) Total strain magnitude.

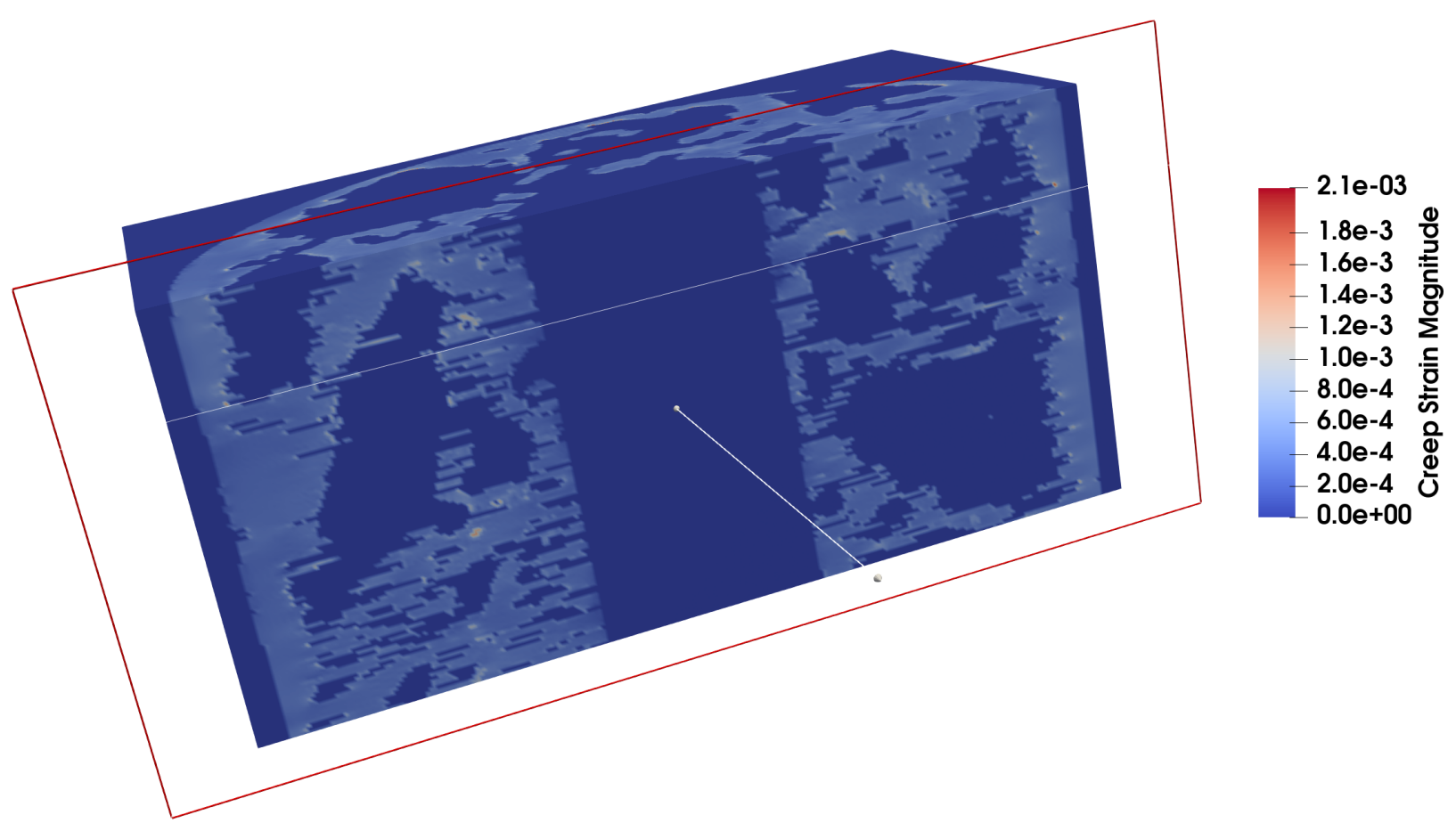

(b) Creep strain magnitude.

Figure 13. Total strain (a) and creep strain (b) in clipped 3D domain at nine days of irradiation. 


\section{LIMITATIONS AND DISCUSSION}

The processes of generating a 3D phase map and using it as a simulation domain involve multiple numerical tools and methods, as detailed in this report. Limitations of this approach are therefore related to different stages of these processes. This section summarizes these limitations and discusses potential improvements to the proposed approach.

- Low contrast: Despite its powerful capability of reconstructing a 3D sample in a nondestructive way, XCT has contrast limitations. When applied to concrete, it is difficult to separate the HCP from the aggregates within the concrete, as a result of their comparable gray levels. Thus, a threshold-based segmentation is quite difficult and would be time-consuming given the number of layers involved in the scan (1,018 layers for the scan used in this work). The unassisted learning algorithm used in this work (DBSCAN) performs well with optimized parameters and after artificially enhancing contrast in the images. However, the overall result still needs improvements; discontinuities were observed along the vertical direction of the 3D domain, as shown in Figure 12a. A possible alternative to DBSCAN is a deep learning algorithm for image segmentation. In this case, manual segmentation of a number of images would be required to train the algorithm to identify the different phases within the images. This technique has been successfully applied to XCT scans with contrast issues, and it has shown good evaluation accuracy [9]. Also, several neural network architectures for semantic segmentation, such as U-Net and SegNet, are available [10,11].

- Image quality: In addition to contrast issues, the low quality of the images does not allow for the identification of separate minerals and particles within aggregates using the approach documented herein. The aggregates that compose the concrete sample used in this work specifically comprise very fine particles. Moreover, some particles show a brighter gray level within an aggregate block in the images. To mitigate this issue, an artificial grain structure is used in this work to create particles within aggregates. However, assisted learning algorithms could also be explored for this problem.

- Grain structure: The Voronoi partition used in this research is applied to the entire 3D domain, including HCP and void phases, despite being needed in the aggregate phase only. It is in fact difficult to restrict the grain sizes to the aggregate phase given its shape, which adds more computational expense to the problem. The issue with this approach is more visible when the particle or grain size is large and comparable to the aggregate size. In this case, the particle distribution over aggregates is biased because the continuation of a grain is observed in other aggregate areas. Therefore, it is recommended to use this technique for small grain sizes to reduce the bias.

- Aggregate composition: In this work, quartz has the largest volume fraction, and other minerals have relatively small volume fractions. This presents a limitation on the accuracy of the aggregate composition because the grains may not be small enough to represent a mineral with a small volume fraction. However, this issue is not expected to significantly affect the mechanical behavior of the simulated concrete. Furthermore, in this work, each grain variable (or order parameter) used in MOOSE to generate the grain structure represents a number of grains in the domain as shown in Figure 8. As previously mentioned, order parameters were used to assign a mineral, instead of individual grains. Another option is to separate individual grains in postprocessing to assign minerals for better composition accuracy.

- Background and void regions: The background and void regions in the XCT images are combined 
into a single phase in this work. This choice is related to the nature of these regions (mostly air). However, their presence in the simulation domain is a limitation because it requires the use of a constitutive model in this phase to maintain the continuity of the mechanical model in the domain. Linear elasticity is employed in regions with artificial elastic properties. The choice of these properties necessitates special care to avoid nonphysical behaviors in the simulation.

- Computational cost: The entire process required to generate the MOSAIC-compatible 3D phase map incurred a relatively low computational cost, as a result of the initial decrease in image resolution. For instance, the image processing part uses a few seconds for each image. The grain structure generation uses approximately four hours with the previously discussed resources and mesh distribution techniques. The gradient-based particle map generation is done within a few minutes. The MOSAIC script that creates ITZ and IPI regions runs in approximately 1.5 to 2 hours. Moreover, the 3D simulation in MOSAIC resulted in 18 time steps (in logarithmic increments) corresponding to approximately 9 days of irradiation in less than 24 hours in wall time using a single core on a 64GB RAM workstation. Two main processing and postprocessing Python scripts are used in this approach: image processing and phase assignment. However, it is important to note that only 1,000 3D grains were generated in this example, which resulted in a relatively large average grain size. As the number of grains increases, the average grain size is reduced, and consequently, the computational cost is expected to go up for certain steps of this process. For instance, to generate more grains in MOOSE, more variables may be needed, which increases the memory cost. 


\section{CONCLUSION}

Following the successful development, application, and validation of the FFT-based code MOSAIC using realistic 2D concrete microstructures, a new approach to construct 3D microstructures based on XCT data is presented in this report. XCT provides information on a specimen in a nondestructive manner based on the different radiodensities of its constituents. This enables the generation of a series of 2D gray scale images representing the layers of the specimen along the scanned volume. An XCT scan of a hollow cylinder concrete specimen was performed, and 1,018 layers of the specimen were obtained. Each image or layer has a resolution of $1008 \times 1024$ pixels. In this work, several image processing techniques were used to process the images and identify the specimen's constituents to produce a realistic 3D simulation domain for concrete. However, the phase identification process is not trivial because of the low contrast between aggregates and the HCP. Additionally, it is difficult to separate minerals within the aggregates because of the low contrast and low image quality. Therefore, several image processing methods are employed to help to address these issues.

The report describes three major steps:

1. A clustering algorithm is applied to the processed images to detect clusters of pixels with similar features and separate the three phases (HCP, aggregates, and pores).

2. The processed images are stacked to form a 3D simulation domain. An FEM-based simulation environment,MOOSE, is used to separate the three phases into mesh blocks and to generate a grain structure using a Voronoi diagram.

3. The data obtained from the FEM code are processed to assign minerals to the newly generated grains inside the aggregate block. Interfaces between particles and between phases are added in MOSAIC, and a full 3D phase map is produced.

An example 3D simulation in MOSAIC of irradiated concrete using linear elasticity, RIVE, and creep models is presented using the $3 \mathrm{D}$ phase map.

Although the phase identification methodology shows good results despite the contrast issue, other methods such as an assisted learning segmentation algorithms can improve the quality of the 3D microstructure. 


\section{REFERENCES}

\section{References}

[1] C.E. Torrence, A.B. Giorla, Y. Li, E. Tajuelo-Rodriguez, J.D. Arregui-Mena, T.M. Rosseel, and Y. Le Pape. Mosaic: An effective fft-based numerical method to assess aging properties of concrete. Journal of Advanced Concrete Technology, 19(2):149-167, 2021.

[2] Ippei Maruyama, Osamu Kontani, Masayuki Takizawa, Shohei Sawada, Shunsuke Ishikawa, Junichi Yasukouchi, Osamu Sato, Junji Etoh, and Takafumi Igari. Development of soundness assessment procedure for concrete members affected by neutron and gamma-ray irradiation. Journal of Advanced Concrete Technology, 15(9):440-523, 2017.

[3] M. Daszykowski and B. Walczak. Density-Based Clustering Methods. Comprehensive Chemometrics, 2:635-654, 2009.

[4] Nadia Rahmah and Imas Sukaesih Sitanggang. Determination of Optimal Epsilon (Eps) Value on DBSCAN Algorithm to Clustering Data on Peatland Hotspots in Sumatra. IOP Conference Series: Earth and Environmental Science, 31(1), 2016.

[5] Derek Gaston, Chris Newman, Glen Hansen, and Damien Lebrun-Grandié. MOOSE: A parallel computational framework for coupled systems of nonlinear equations. Nuclear Engineering and Design, 239(10):1768-1778, 2009.

[6] J Ahrens, Geveci B, and C Law. ParaView: An End-User Tool for Large Data Visualization ParaViewWeb View project. 836, 2005.

[7] Y. Le Pape, M.H.F. Alsaid, and A.B. Giorla. Rock-forming minerals radiation-induced volumetric expansion - revisiting the literature data. Journal of Advanced Concrete Technology, 16:191-209, 2018.

[8] Y. Li, Y. Le Pape, E. , Tajuelo Rodriguez, C.E. Torrence, J.D. Arregui-Mena, T.M. Rosseel, and M. Sircar. Microstructural characterization and assessment of mechanical properties of concrete based on combined elemental analysis techniques and Fast-Fourier transform - based simulations. Construction and Building Materials, 257, Oct 2020.

[9] Aly Badran, David Marshall, Zacharie Legault, Ruslana Makovetsky, Benjamin Provencher, Nicolas Piché, and Mike Marsh. Automated segmentation of computed tomography images of fiber-reinforced composites by deep learning. Journal of Materials Science, 55(34):16273-16289, 2020.

[10] O Ronneberger, P Fischer, and T Brox. Medical Image Computing and Computer-Assisted Intervention - MICCAI 2015: 18th International Conference Munich, Germany, October 5-9, 2015 proceedings, part III. Lecture Notes in Computer Science (including subseries Lecture Notes in Artificial Intelligence and Lecture Notes in Bioinformatics), 9351(Cvd):12-20, 2015.

[11] Vijay Badrinarayanan, Alex Kendall, and Roberto Cipolla. SegNet: A Deep Convolutional EncoderDecoder Architecture for Image Segmentation. IEEE Transactions on Pattern Analysis and Machine Intelligence, 39(12):2481-2495, 2017. 